\title{
Compatibility Conditions in Continua with Microstrusture
}

\author{
Evgenii V. Murashkin ${ }^{1,2,3}$ and Nikita E. Stadnik ${ }^{1,4}$ \\ ${ }^{1}$ Institute for Problems in Mechanics of the Russian Academy of Sciences, Vernadsky Ave 101 Bldg 1, Moscow, 119526, Russia \\ ${ }^{2}$ Bauman Moscow State Technical University, 2nd Baumanskaya Str 5/1, Moscow, 105005, Russia \\ ${ }^{3}$ National Research Nuclear University T«MEPhITi, Kashirskoye shosse 31, Moscow, 115409, Russia \\ ${ }^{4}$ Moscow Technological University, Vernadsky Ave 78, Moscow, 119454, Russia
}

\begin{abstract}
The present study is devoted to the statement of compatibility conditions on propagating wave sur- faces of strong and weak discontinuities in thermoelastic continua with microstructure. The field formalism is used to study the problem. A natural density of thermoelastic action and the corresponding variational least action principle are stated for a varying domain. A special form of the first variation of the action is employed to obtain 4-covariant boundary conditions on the wave surfaces. These are given by jumps of the Piola-Kirchhoff stress 4-tensor and the energy-momentum tensor. Problems of propagation of weak discontinuities in type-II MPTE continua are considered. Geometrical and kinematical compatibility conditions are used to study possi- ble wave surfaces of weak discontinuities. It is shown that the surfaces of weak discontinuities can propagate without weak discontinuities of the temperature displacement.
\end{abstract}

\section{Introduction}

Problems of micropolar continua take its origin from the classical E. and F. Cosserat's paper [1]. Micropolar (MP) continuum theories include not only translational displacements but also additional degrees of freedom. These degrees of freedom are coupled with changes in reper (three directors) associated with microvolume. Such changes may be described by a rotation vector when reper associated with microvolume are rigid rotated. In contrary to conventional elasticity a continuum with microstucture is described by the asymmetric strain and stress tensors known from many previous discussions [2, $3,6]$. Thus the asymmetric elastic theory is characterized by a comparatively large number of constitutive elastic constants need to be determined from the experimental observations. There are several phenomena (for example, additive manufacturing design $[4,5]$, biomechanics, nematic liquid crystals [7, 8] behavior, the anomalous piezoelectric effect in quartz, the dispersion of elastic waves, as well as a number of other experimentally observed elastic properties of the pure crystals) being beyond the scope of the conventional thermoelasticity (CTE) and piezoelectroelasticity, the finite propagation velocity of thermal waves known as second sound waves.

The phenomenon of the second sound is observed at very low temperatures in crystals of high purity. The observation of the second sound in the crystal $\mathrm{NaF}$ is discussed (see [9]). The second sound in the $\mathrm{NaF}$ is observed at a temperature of about $15 K$ [10]. The existence of second sound waves was also confirmed in the NaF (see [11] and [12]); the speed of the second sound waves is $1953.1 \mathrm{~m} / \mathrm{S}$ see [13]. In the publication [14] the measurements of the second sound waves in bismuth (Bi) at a temperature of about $3 K$ are described. The speed of the second sound waves in the bismuth is equal to $78 \pm 5 \mathrm{~m} / \mathrm{S}$.

The micropolar thermoelastic (MPTE) continuum may be described in terms of field formalism, for example, from positions of the Green-Naghdi thermoelasticity (GN-theory) $[15,16]$. Now such mathematical frame- works of the thermoelastic behavior of solids are rapidly refined [17-20]. They are based on different modifications of the classical Fourier law of heat conduction. GN- thermoelasticity theory itself can be subdivided into the three different types. Type-I theory (GNI/CTE thermoelasticity) is based on the classical Fourier law of heat conduction with an infinite velocity of propagation of an exponentially decaying heat signal. Type-II theory (GNII, hyperbolic thermoelasticity) is characterised by the energy conservation and the finite propagation velocity of thermal waves known as second sound waves. Type-III theory (GNIII) combines both type-I and type-II thermoelasticity. Thereby type-I and type-II theories are the limiting cases of type-III theory. GNIII-theory obviously encircles a wider range of phenomena, as compared with the classical Fourier heat conduction (CTE) theory.

\section{Compatibility conditions for jumps}

The mathematical models are to simultaneously fulfill the following frameworks: 1.) Finiteness of the heat signal propagation velocity, and 2.) The ability of the spatial 
propagation of the thermoelastic waves without attenuation, and 3.) Existence of distortionless wave forms akin to the classical d'Alembert type waves.

A field theory formalism involves mathematical description of physical fields by integral action functional. A general form of action within a variable domain of 4spacetime with the elementary volume of $d^{4} X=d X^{1} d X^{2} d X^{3} d X^{4}$ is

$$
\mathfrak{I}=\int L\left(X^{\beta}, \varphi^{k}, \partial_{\alpha} \varphi^{k}\right) d^{4} X
$$

where $\varphi^{k}$ is the physical fields array, $L$ is the Lagrangian density.

The least action principle states that the actual field is realized in the spacetime in a way that the action of (1) is minimum, i.e. for any admissible variations of physical fields $\varphi^{k}$ and non-variable coordinates $X^{\beta}$ are $\delta \mathfrak{I}=0$. Then the classical Euler-Lagrange equations are held:

$$
E_{k}(L)=\frac{\partial L}{\partial \varphi^{k}}-\partial_{\beta} \frac{\partial L}{\partial\left(\partial_{\beta} \varphi^{k}\right)}=0 .
$$

In general, a conservation law has the following form

$$
\partial_{\beta} J^{\beta}=0
$$

where the vector $J^{\beta}$ is the generalized vector 4-current. By finite variation $\delta^{\nabla}=\delta / \varepsilon$ the 4-current can be obtained as

$$
J^{\beta}=\frac{\partial L}{\partial\left(\partial_{\beta} \varphi^{k}\right)} \delta^{\nabla} \varphi^{k}+\left(L \delta_{\alpha}^{\beta}-\left(\partial_{\alpha} \varphi^{k}\right) \frac{\partial L}{\partial\left(\partial_{\beta} \varphi^{k}\right)}\right) \delta^{\nabla} X^{\alpha},
$$

if the variational symmetries of the action are known from previous considerations.

The finite variations of action is (see [21])

$$
\delta^{\nabla} \mathfrak{I}=\int \partial_{\beta} J^{\beta} d^{4} X
$$

Assuming the temperature field is continuous and temperature gradient of the first order can be discontinuous by passing through some bilateral surface $\Sigma^{ \pm}$propagating with the normal velocity $G$ and normal unit 4-vector $N_{\beta}$ in 4-spacetime.

We replace the integral over 4-volume in equation (3) on sum of surface integrals. Then only two surface integrals over surface $\Sigma$ are remained when the variations $\delta^{\nabla} \varphi^{k}$ and $\delta^{\nabla} X^{\alpha}$ are fixed on the outer boundary of the field:

$$
\delta \mathfrak{I}=\oint_{\Sigma^{+}} J^{\beta} N_{\beta} d^{3} \tau-\oint_{\Sigma^{-}} J^{\beta} N_{\beta} d^{3} \tau=\oint_{\Sigma}\left[J^{\beta}\right] N_{\beta} d^{3} \tau .
$$

Hereafter square brackets denote the jumps.

The equation $\delta \mathfrak{I}=0$ is valid for the actual field and a variations of $\delta^{\nabla} \varphi^{k}$ and $\delta^{\nabla} X^{\alpha}$ are continuous in passing through the surface $\Sigma$. Therefore following 4-covariant compatibility conditions for strong discontinuities is obtained from equation (4):

$$
\begin{gathered}
N_{\beta}\left[-\frac{\partial L}{\partial\left(\partial_{\beta} \varphi^{k}\right)}\right]=0, \\
N_{\beta}\left[L \delta_{\alpha}^{\beta}-\left(\partial_{\alpha} \varphi^{k}\right) \frac{\partial L}{\partial\left(\partial_{\beta} \varphi^{k}\right)}\right]=0 .
\end{gathered}
$$

One can see, that the compatibility conditions (5) on strong discontinuities surfaces contain the jumps of the energy-momentum 4-tensor and the Piola-Kirchhoff 4tensor

$$
\begin{gathered}
S_{\cdot \cdot k}^{\beta \cdot}=-\frac{\partial L}{\partial\left(\partial_{\beta} \varphi^{k}\right)}, \\
T_{\cdot \alpha}^{\beta \cdot}=L \delta_{\alpha}^{\beta}-\left(\partial_{\alpha} \varphi^{k}\right) \frac{\partial L}{\partial\left(\partial_{\beta} \varphi^{k}\right)} .
\end{gathered}
$$

The compatibility conditions for jumps of the energymomentum 4-tensor in view of (4) is rewritten in form

$$
\left[T_{\cdot \alpha}^{\beta \cdot}\right] N_{\beta}=[L] N_{\alpha}+\left[\partial_{\alpha} \varphi^{k} S_{\cdot k}^{\beta \cdot}\right] N_{\beta}=0
$$

Equations (7) can be transformed by the compatibility conditions of the strong discontinuities of the PiolaKirchhoff 4-tensor into

$$
[L] N_{\alpha}+\left[\partial_{\alpha} \varphi^{k}\right] S_{\cdot k}^{\beta \cdot} N_{\beta}=0 .
$$

The only significant equation from the compatibility conditions for jumps of the energy-momentum 4-tensor by using the Hadamard-Thomas geometric first order compatibility conditions [22] $\left[\partial_{\alpha} \varphi^{k}\right]=N_{\alpha} F^{k}$ is obtained

$$
[L] N_{\alpha}+S_{\cdot k}^{\beta \cdot} N_{\beta} F^{k}=0 .
$$

The three-dimensional form of the compatibility conditions on the surface of strong discontinuity of the field is derived from the resulting 4-covariant form (4):

$$
\begin{gathered}
{\left[S_{\cdot k}^{\beta \cdot}\right] n_{\mu}=0} \\
{[L]+\left(-G S_{\cdot k}^{4 \cdot}+S_{\cdot k}^{\mu \cdot} n_{\mu}\right)\left(-G\left[\partial_{4} \varphi^{k}\right]+n_{\mu}\left[\partial_{\mu} \varphi^{k}\right]\right)=0}
\end{gathered}
$$

Hereafter $n_{\mu}$ stand for normal unit 3-vector, $\lambda, \mu=1,2,3$.

The compatibility conditions for strong discontinuities are complemented by well known three-dimensional geometrical and kinematic Hadamard-Thomas compatibility conditions of the first and the second order [22] valid for an arbitrary field $\varphi^{k}$

$$
\begin{gathered}
{\left[\partial_{i} \partial_{j} \varphi^{k}\right]=C^{k} n_{i} n_{j}+g^{\alpha \beta} \partial_{\alpha^{\prime}} B^{k}\left(n_{i} \partial_{\beta^{\prime}} x_{j}+n_{j} \partial_{\beta^{\prime}} x_{i}\right)-} \\
-g^{\alpha \beta} g^{\sigma \tau} B^{k} b_{\alpha \sigma} \partial_{\beta^{\prime}} x_{i} \partial_{\tau^{\prime}} x_{j}, \\
{\left[\partial_{i} \partial_{4} \varphi^{k}\right]=\left(-C^{k} G+\delta_{4} B^{k}\right) n_{i}+g^{\alpha \beta} \partial_{\alpha^{\prime}}\left(B^{k} G\right) \partial_{\beta^{\prime}} x_{i},}
\end{gathered}
$$




$$
\begin{aligned}
{\left[\partial_{4} \partial_{4} \varphi^{k}\right] } & =\left(C^{k} G-\delta_{4} B^{k}\right) G+B^{k} \delta_{4} G, \\
{\left[\partial_{4} \varphi^{k}\right] } & =-B^{k} G, \quad\left[\partial_{i} \varphi^{k}\right]=B^{k} n_{i},
\end{aligned}
$$

where $i, j=1,2,3 ; \quad \alpha, \beta, \tau, \sigma=1,2$; the prime in superscripts are denoted by the Greek indices related to surface Gaussian coordinates; $D=\left[\partial_{i} \partial_{j} \varphi\right] n^{i} n^{j} ; \delta_{4}$ is the delta-derivative operator. Those are also due to Rankine and Hugoniot [23, 24].

\section{Action for micropolar continua}

The theory GNII is the only thermodynamically correct theory which satisfies the principles listed in Sec. 1. The action taking into account of the polar microstructure can be adopted in the following form [21], with following field variables: $X^{\alpha} \quad(\alpha=1,2,3)$ are the Lagrangian coordinates; $x^{j}(j=1,2,3)$ are the Eulerian coordinates; $d^{j}(a=1,2,3)$ are micropolar directors associated with microvolume; $\vartheta$ is the thermal displacement field coupled with temperature $\theta$ by equation $(\theta=\dot{\vartheta})$.

Action density (1) we define in the form

$$
\begin{aligned}
& L=\frac{1}{2}\left(\partial_{4} x^{k}\right) \rho_{k j}\left(\partial_{4} x^{j}\right)+\frac{1}{2}\left(\partial_{4} \underset{a}{d^{i}}\right) \stackrel{a b}{i j}_{i j}\left(\partial_{4} d_{b}^{j}\right)- \\
& -\psi\left(X^{\alpha}, x^{j}, \underset{a}{d^{j}}, \vartheta, \partial_{4} \vartheta, \partial_{\alpha} x^{j}, \partial_{\alpha} \underset{a}{d^{j}}, \partial_{\alpha} \vartheta\right) .
\end{aligned}
$$

wherein $\stackrel{a b}{I} I_{i j}$ is the microinertion tensor; $\rho_{i j}$ is the mass density tensor; $\psi$ is the volume density of the Helmholtz's free energy. For tensors $\stackrel{a b}{I}_{i j}$ and $\rho_{i j}$ the symmetry conditions $\stackrel{a b}{I} I_{i j}=I_{j i}^{a b}, \rho_{i j}=\rho_{j i}$ are stated.

The field equations in this case read:

$$
\begin{aligned}
& \partial_{\alpha} S_{\cdot j}^{\alpha \cdot}-\partial_{4} P_{j}=-\frac{\partial L}{\partial\left(x^{j}\right)} \quad(\alpha=1,2,3 ; j=1,2,3), \\
& \partial_{\alpha}{ }^{a} M_{\cdot j}^{\alpha \cdot}+{ }^{a} A_{j}-\partial_{4} Q_{j}=0 \quad(a=1,2,3 ; \alpha=1,2,3 ; j=1,2,3), \\
& \partial_{\alpha} j_{R}^{\alpha}+\partial_{4} s=-\frac{\partial L}{\partial \vartheta} \quad(\alpha=1,2,3),
\end{aligned}
$$

and are supplemented by the constitutive equations:

$$
\begin{aligned}
& \partial_{\alpha} S_{\cdot j}^{\alpha \cdot}=-\frac{\partial L}{\partial\left(\partial_{\alpha} x^{j}\right)}, \quad P_{j}=\frac{\partial L}{\partial\left(\partial_{4} x^{j}\right)} \\
& \partial_{\alpha}{ }^{a} M_{\cdot j}^{\alpha \cdot}=-\frac{\partial L}{\partial\left(\partial_{\alpha} d^{j}\right)}, \quad{ }_{a}^{a} A_{j}=\frac{\partial L}{\partial d^{j}}, \quad \partial_{4} Q_{j}=\frac{\partial L}{\partial\left(\partial_{4} d^{j}\right)}, \\
& \partial_{\alpha} j_{R}^{\alpha}=\frac{\partial L}{\partial\left(\partial_{\alpha} \vartheta\right)}, \quad s=\frac{\partial L}{\partial\left(\partial_{4} \vartheta\right)},
\end{aligned}
$$

In this notation the energy-momentum 4-tensor is rewritten in following forms

$$
\begin{aligned}
& T_{\cdot \lambda}^{\mu \cdot}=L \delta_{\lambda}^{\mu}-S_{l}^{\mu} \partial_{\lambda} x^{l}-M_{\cdot j}^{\mu \cdot} \partial_{\lambda} d_{\mathrm{a}}^{l}-j_{R}^{\mu} \partial_{\lambda} \vartheta=P_{\lambda}^{\mu}, \\
& T_{\cdot 4}^{\mu \cdot}=-S_{l}^{\mu} \partial_{4} x^{l}-M_{\cdot j}^{\mu \cdot} \partial_{4} d_{\mathrm{a}}^{l}-j_{R}^{\mu} \partial_{4} \vartheta=\Gamma_{\mu}, \\
& T_{\cdot \lambda}^{4 \cdot}=-P_{l} \partial_{\lambda} x^{l}-Q_{l} \partial_{\lambda} d_{\mathrm{a}}^{l}-s \partial_{\lambda} \vartheta=P_{\lambda}, \\
& T_{\cdot 4}^{4 \cdot}=\mathrm{L}-P_{l} \partial_{4} x^{l}-Q_{l} \partial_{4} d_{\mathrm{a}}^{l}-s \partial_{4} \vartheta=-H,
\end{aligned}
$$

Here, well known in theoretical physics characteristics, $P_{\lambda}^{\mu}$ is the Eshelby stress tensor, $\Gamma_{\mu}$ is the Umov-Poynting vector, $P_{\lambda}$ is pseudo-momentum vector, $H$ is quasidensity of the Hamiltonian.

Compatibility conditions on strong discontinuity surface propagating in MPTE-II continuum, according to eqs. (9), (10), take the forms:

$$
\begin{aligned}
& \frac{1}{2}\left[\left(\partial_{4} x^{k}\right) \rho_{k j}\left(\partial_{4} x^{j}\right)\right]+\frac{1}{2}\left[\left(\partial_{4} d_{a}^{i}\right) \underset{i j}{a b}\left(\partial_{4} d_{b}^{j}\right)\right]- \\
& -\left[\psi\left(X^{\alpha}, x^{j}, \underset{a}{d^{j}}, \vartheta, \partial_{4} \vartheta, \partial_{\alpha} x^{j}, \partial_{\alpha} d_{a}^{j}, \partial_{\alpha} \vartheta\right)\right]+ \\
& +\left(G I_{l k}^{a b} \partial_{4} d_{b}^{l}-n_{\mu}{ }^{a} M_{\cdot k}^{\mu \cdot}\right)\left(G\left[\partial_{4} d_{b}^{k}\right]-n_{\mu}\left[\partial_{\mu} d_{b}^{k}\right]\right)+ \\
& +\left(G \rho_{l k} \partial_{4} x^{l}-n_{\mu} S_{\cdot k}^{\mu \cdot}\right)\left(G\left[\partial_{4} x^{k}\right]-n_{\mu}\left[\partial_{\mu} x^{k}\right]\right)+ \\
& +\left(G s-n_{\mu} j_{R}^{\mu \cdot}\right)\left(G\left[\partial_{4} \vartheta\right]-n_{\mu}\left[\partial_{\mu} \vartheta\right]\right), \\
& G \rho_{k l}\left[\partial_{4} x^{k}\right]=n_{\mu}\left[S_{\cdot l}^{\mu \cdot}\right], \\
& G I_{k l}^{a b}\left[\partial_{4} d_{b}^{k}\right]=n_{\mu}\left[{ }^{a} M_{\cdot l}^{\mu \cdot}\right], \\
& G[s]=n_{\mu}\left[j_{R}^{\mu \cdot}\right] .
\end{aligned}
$$

\section{Governing equations of GNII continuum. Linear case}

A mathematical model of MPTE-II continuum (see [2] for details) is used throughout the paper. Such continua permit the existence of rotational degrees of freedom (a rotation vector $\varphi$ ) along with translational degrees of freedom (the translational displacement vector $\mathbf{u}$ ). Thus the natural action density may be accepted in form

$$
L=\frac{1}{2} u \cdot \boldsymbol{\rho} \cdot u+\frac{1}{2} \dot{\boldsymbol{\varphi}} \cdot \boldsymbol{J} \cdot \dot{\boldsymbol{\varphi}}-\psi(\boldsymbol{e}, \boldsymbol{\Gamma}, \theta, \nabla \vartheta),
$$

where $\boldsymbol{\rho}$ is the mass density tensor, $\mathrm{J}$ is a tensor of inertia, $\psi$ is the free energy per unit of volume, $\theta$ is the actual temperature, $\nabla$ is the three-dimensional Hamiltonian differential operator (the nabla symbol), dot over a symbol denotes partial differentiation with respect to time at fixed spatial coordinates.

The asymmetric strain tensor $\boldsymbol{e}$ and bending-torsion tensor $\boldsymbol{\Gamma}$ are associated with translational displacements $u$ and microrotations $\varphi$ by formulae

$$
\begin{aligned}
& \boldsymbol{e}=\nabla \otimes u-\boldsymbol{\varepsilon} \cdot \boldsymbol{\varphi}, \\
& \boldsymbol{\Gamma}=\nabla \otimes \boldsymbol{\varphi},
\end{aligned}
$$


The equation (17) are represented in a rectangular coordinate system as

$$
\begin{aligned}
& e_{j i}=\partial_{j} u_{i}-\varepsilon_{j i k} \phi_{k}, \\
& \Gamma_{j i}=\partial_{j} \phi_{i} .
\end{aligned}
$$

For MPTE-II continuum, equations of motion of micropolar medium are written (according to eq. (13)) in direct tensor representation for the case of the absence of mass forces and mass moments

$$
\left\{\begin{array}{l}
\nabla \cdot \boldsymbol{\sigma}=\boldsymbol{\rho} u, \\
\nabla \cdot \boldsymbol{m}+\boldsymbol{\varepsilon} \cdot \boldsymbol{\sigma}=\mathrm{J} \ddot{\boldsymbol{\varphi}} .
\end{array}\right.
$$

where $\boldsymbol{\varepsilon}$ is the three-dimensional Levi-Civita symbol (permutation symbol, antisymmetric symbol, or alternating symbol).

Equations (19) in rectangular co-ordinate system can be deduced in form

$$
\left\{\begin{array}{l}
\partial_{j} \sigma_{j i}=\rho_{i j} \ddot{u}_{j} \\
\partial_{j} m_{j i}+\varepsilon_{i j k} \sigma_{j k}=J_{i j} \ddot{\phi}_{j}
\end{array}\right.
$$

The entropy balance equation are taken in form

$$
\dot{s}=-\nabla \cdot \dot{j}_{R},
$$

where $s$ denotes the entropy referred to the unit of volume, $\boldsymbol{j}_{R}$ is the entropy flux vector.

The Clausius-Duhem inequality is satisfied by using the law of heat conduction, which states the proportionality of the heat velocity flux vector $\boldsymbol{h}$ and negative spatial temperature gradient $\theta$

$$
\dot{\boldsymbol{h}}=-\boldsymbol{\Lambda} \cdot \nabla \theta
$$

where $\boldsymbol{\Lambda}$ is the material thermal conductivity tensor (thermal diffusion tensor). $\boldsymbol{\Lambda}$ is a positive definite matrix.

Since the free energy $\psi(\boldsymbol{e}, \boldsymbol{\Gamma}, \theta, \nabla \vartheta)$ is the function of the independent variables $\boldsymbol{e}, \boldsymbol{\Gamma}, \theta, \nabla \vartheta$ then the constitutive equations (14) can be rewritten

$$
\begin{array}{ll}
\sigma_{j i}=\frac{\partial \psi}{\partial e_{j i}}, & \mu_{j i}=\frac{\partial \psi}{\partial \Gamma_{j i}}, \\
s=-\frac{\partial \psi}{\partial \theta}, & \xi+h \cdot \frac{\nabla \theta}{\theta^{2}}=0 .
\end{array}
$$

Let us expand the free energy $\psi$ into the Taylor series in the vicinity of the natural state $\boldsymbol{e}=\Gamma=0, \theta=0$, disregarding the terms of higher order than the second one. The following form of the expansion is obtained for isotropic, homogeneous and centrosymmetric bodies

$$
\begin{aligned}
& \psi=\frac{\mu+\eta}{2} e_{j i} e_{j i}+\frac{\mu-\eta}{2} e_{j i} e_{i j}+\frac{\lambda}{2} e_{k k} e_{n n}+ \\
& +\frac{\gamma+\varepsilon}{2} \Gamma_{j i} \Gamma_{j i}+\frac{\gamma-\varepsilon}{2} \Gamma_{j i} \Gamma_{i j}++\frac{\beta}{2} \Gamma_{k k} \Gamma_{n n}- \\
& -\alpha e_{k k} \theta-\varsigma \Gamma_{k k} \theta-\frac{\Lambda}{2} \theta^{2} .
\end{aligned}
$$

Therefore the constitutive equations of MPTE-II continuum can be derived according to equations (22) and (23)

$$
\begin{aligned}
& \sigma_{i j}=(\mu+\eta) e_{j i}+(\mu-\eta) e_{i j}+\left(\lambda \operatorname{tr} e_{k k}-\alpha \theta\right) \delta_{i j}, \\
& m_{i j}=(\gamma+\varepsilon) \Gamma_{j i}+(\gamma-\varepsilon) \Gamma_{i j}+\left(\beta \operatorname{tr} \Gamma_{k k}-\varsigma \theta\right) \delta_{i j}, \\
& s=\alpha e_{k k}+\varsigma \Gamma_{k k}+\Lambda \theta, \\
& j_{\mathrm{R}}=\kappa \nabla \vartheta .
\end{aligned}
$$

wherein $\lambda, \mu, \eta, \gamma, \beta, \varepsilon$ are isothermal constitutive constants of type-II micropolar thermoelastic (GNII) continuum; $\alpha, \varsigma$ are constitutive constants providing coupling of equations of motion and heat conduction; $\Lambda$ is the material thermal conductivity (thermal diffusion); $\kappa$ is the heat capacity (per unit volume) at constant (zero) strains. Constants $\alpha, \varsigma$ depend not only on the mechanical properties of the continuum, but also depend on the thermal properties.

Consequently the hyperbolic heat conduction equation can be derived by transforming equations (21) and (24) as

$$
\Lambda \cdot \nabla^{2} \vartheta-\kappa \ddot{\theta}-\alpha \nabla \cdot \dot{\boldsymbol{u}}-\varsigma \nabla \cdot \dot{\boldsymbol{\varphi}}=0 .
$$

After substituting the stress tensors $\boldsymbol{\sigma}$ and $\boldsymbol{m}$ from the formulae (24) in the equations of motion (19) and taking account of (17) the system of coupled partial differential equations of motion and heat conduction for a linear isotropic GNII continuum in the absence of mass forces, moments, and heat sources can be written as $[2,6]$ :

$\left\{\begin{array}{l}(\lambda+\mu-\eta) \nabla \nabla \cdot u+(\mu+\eta) \nabla^{2} u+2 \eta \nabla \times \varphi-\alpha \nabla \dot{\vartheta}-\rho u=0, \\ (\beta+\gamma-\varepsilon) \nabla \nabla \cdot \varphi+(\gamma+\varepsilon) \nabla^{2} \varphi-4 \eta \varphi+2 \eta \nabla \times u-\varsigma \nabla \dot{\vartheta}-\mathrm{J} \ddot{\varphi}=0, \text { (26) } \\ \nabla^{2} \vartheta-\frac{\kappa}{\Lambda} \ddot{\vartheta}-\frac{\alpha \theta_{0}}{\Lambda} \nabla \cdot u-\frac{\varsigma \theta_{0}}{\Lambda} \nabla \cdot \dot{\varphi}=0 .\end{array}\right.$

Hereafter $\theta$ is the temperature increment over the referential temperature.

As pointed out the nonzero constitutive constants $\alpha$, $\varsigma$ provide coupling of micropolar thermoelasticity equations. It is usually assumed that $\varsigma=0$ (see [2]). This constitutive constant is kept in all further considerations for the completeness analysis.

The scalar equation in the system (26) is called a gen eralized heat conduction equation conjugate to the equations of motion (the first and the second equations in (26)).

\section{Propagating weak discontinuities of thermomechanical fields in MPTE-II continuum}

This section deals with the processes of propagating weak discontinuities of translational displacements $\mathbf{u}$, microrotations $\varphi$ and temperature $\theta$ in the MPTE-II continuum. System of partial differential equations (26) includes partial derivative order not higher than the second. Let a wavefront (wave surface $\Sigma$ ) of weak discontinuities displacements $\boldsymbol{u}$, microrotations $\varphi$ and temperature $\theta$ be propagated in three-dimensional space 
with normal velocity $G$. Unit normal vector to that wave surface is denoted by $\mathbf{n}$. If surface $\Sigma$ is parameterized by

$$
x^{i}=\psi^{i}\left(y^{1}, y^{2}, t\right)
$$

Then components of first $g^{\alpha \beta}$ and second $b_{\alpha \beta}$ fundamental forms of surface $\Sigma$ are computed by formulas

$$
\begin{aligned}
& g_{\alpha \beta}=\frac{\partial \psi^{i}}{\partial y^{\alpha}} \frac{\partial \psi^{i}}{\partial y^{\beta}}=\partial_{\alpha^{\prime}} x^{i} \partial_{\beta^{\prime}} x^{i}, \\
& b_{\alpha \beta}=\frac{\partial^{2} \psi^{i}}{\partial y^{\alpha} \partial y^{\beta}} n^{i}=\partial_{\alpha^{\prime}} \partial_{\beta^{\prime}} x^{i} n^{i}, \\
& \partial_{\alpha} n^{i}=-g^{\beta \gamma} b_{\beta \alpha} \partial_{\gamma^{\prime}} x^{i} .
\end{aligned}
$$

Therefore, kinematical and geometrical compatibility conditions of the second and the first order according to eqs. (11) and (27) are read

$$
\begin{array}{ll}
{[\nabla \otimes \nabla \otimes \boldsymbol{u}]=\boldsymbol{n} \otimes \boldsymbol{n} \otimes \boldsymbol{A},} & {[\nabla \otimes \nabla \otimes \boldsymbol{\varphi}]=\boldsymbol{n} \otimes \boldsymbol{n} \otimes \boldsymbol{S},} \\
{[\nabla \otimes \boldsymbol{u}]=-G \boldsymbol{n} \otimes \boldsymbol{A},} & {[\nabla \otimes \dot{\boldsymbol{\varphi}}]=-G \boldsymbol{n} \otimes \boldsymbol{S},} \\
{[\boldsymbol{u}]=G^{2} \boldsymbol{A},} & {[\ddot{\boldsymbol{\varphi}}]=G^{2} \boldsymbol{S},} \\
{[\nabla \otimes \nabla \vartheta]=B \boldsymbol{n} \otimes \boldsymbol{n},} & {[\ddot{\vartheta}]=G^{2} B,} \\
{[\nabla \otimes \dot{\vartheta}]=-G B \boldsymbol{n},} &
\end{array}
$$

$B, A, S$ are fields defined on this surface, and the equalities $B=0, \boldsymbol{A}=\boldsymbol{0}, \boldsymbol{S}=\boldsymbol{0}$ cannot be satisfied simultaneously at any point of the surface, if the surface is indeed the surface of weak discontinuities. Equations (26) and (28) give the following relations between the jumps of partial derivatives of the second order across the wave surface:

$$
\left\{\begin{array}{l}
\left(\rho G^{2}-(\mu+\eta)\right) \boldsymbol{A}-(\lambda+\mu-\eta) \boldsymbol{n}(\boldsymbol{n} \cdot \boldsymbol{A})-\alpha G B \boldsymbol{n}=0, \\
\left(\mathrm{~J} G^{2}-(\gamma+\varepsilon)\right) \boldsymbol{S}-(\beta+\gamma-\varepsilon) \boldsymbol{n}(\boldsymbol{n} \cdot \boldsymbol{S})-\varsigma G B \boldsymbol{n}=0, \\
B-\frac{\kappa}{\Lambda} G^{2} B+\frac{\alpha G}{\Lambda} \boldsymbol{n} \cdot \boldsymbol{A}+\frac{\varsigma G}{\Lambda} \boldsymbol{n} \cdot \boldsymbol{S}=0 .
\end{array}\right.
$$

The polarization vectors of the wave $\boldsymbol{A}, \boldsymbol{S}$ can be splited into a sum of projections onto the tangent plane and on the normal direction to the wave surface:

$$
\begin{aligned}
& A=A_{\perp} \boldsymbol{\tau}+A_{\|} \boldsymbol{n}, \quad S=S_{\perp} \boldsymbol{\tau}+S_{\|} \boldsymbol{n}, \\
& A_{\perp}=\boldsymbol{A} \cdot \boldsymbol{\tau}, \quad A_{\|}=\boldsymbol{A} \cdot \boldsymbol{n}, \\
& S_{\perp}=\boldsymbol{S} \cdot \boldsymbol{\tau}, \quad S_{\|}=\boldsymbol{S} \cdot \boldsymbol{n} .
\end{aligned}
$$

where $\boldsymbol{\tau}$ is the tangential unit vector and $\boldsymbol{n}$ is the normal unit one of the wave surface respectively. Taking account of equations (30) the system (29) is transformed into

$$
\left\{\begin{array}{l}
\left(\rho G^{2}-(\mu+\eta)\right)\left(A_{\perp} \boldsymbol{\tau}+A_{||} \boldsymbol{n}\right)-(\lambda+\mu-\eta) A_{||} \boldsymbol{n}-\alpha G B \boldsymbol{n}=0, \\
\left(\mathrm{~J} G^{2}-(\gamma+\varepsilon)\right)\left(S_{\perp} \boldsymbol{\tau}+S_{||} \boldsymbol{n}\right)-(\beta+\gamma-\varepsilon) S_{||} \boldsymbol{n}-\varsigma G B \boldsymbol{n}=0, \\
B-\frac{\kappa}{\Lambda} G^{2} B+\frac{\alpha G}{\Lambda} A_{||}+\frac{\varsigma G}{\Lambda} S_{||}=0 .
\end{array}\right.
$$

After rearrangements in equations (31) in view of (30) the following system is derived:

$$
\begin{aligned}
& \left\{\begin{array}{l}
\left(\rho G^{2}-(\mu+\eta)\right) A_{\perp}=0, \\
\left(\rho G^{2}-(\lambda+2 \mu)\right) A_{\mathrm{P}}-\alpha G B=0,
\end{array}\right. \\
& \left\{\begin{array}{l}
\left(\mathrm{J} G^{2}-(\gamma+\varepsilon)\right) S_{\perp}=0, \\
\left(\mathrm{~J} G^{2}-(\gamma+2 \beta)\right) S_{\mathrm{P}}-\varsigma G B=0,
\end{array}\right.
\end{aligned}
$$

The third equation in (31) allows us to couple a weak discontinuity of temperature displacement with a normal projection of weak discontinuities of translational displacements and microrotations by

$$
B=G\left(\alpha A_{\|}+\varsigma S_{\|}\right) \cdot\left(\Lambda-\kappa G^{2}\right)^{-1} .
$$

This new equation is obtained by restrictions $\varsigma \neq 0$.

We proceed to consider the discriminated cases separately. Evidently in the case $\boldsymbol{A}=\mathbf{0}, \boldsymbol{S}=\mathbf{0}$ and returning to system (29) the scalar equation in (29) is satisfied identically, so the surface $\Sigma$ is actually not a surface of weak discontinuities.

The propagation of transverse waves in the MPTE-II continuum is possible in the following cases:

$$
\begin{aligned}
& \text { I case }-A_{\perp} \neq 0, S_{\perp}=0 ; \\
& \text { II case - } A_{\perp}=0, S_{\perp} \neq 0 ; \\
& \text { III case - } A_{\perp} \neq 0, S_{\perp} \neq 0 ; \\
& \text { IV case - } A_{\perp}=0, S_{\perp}=0 .
\end{aligned}
$$

In the first case a weak discontinuity of translational displacements exists only on the surface propagating with the velocity

$$
G=c_{\perp}^{\mu},
$$

where

$$
c_{\perp}^{\mu}=\sqrt{\frac{\mu+\eta}{\rho}} .
$$

It can be elucidated that the velocity $G$ (34) exactly coincides with the one of transverse elastic wave if the constitutive micropolar constant $\eta$ equals to zero $c_{\perp}=\sqrt{\mu / \rho}$.

In the second case, the third equation of the system (32) allows to compute the propagation velocity of a weak discontinuities of microrotation

$$
G=c_{\perp}^{\mu \mu},
$$

where

$$
c_{\perp}^{\mu \mu}=\sqrt{\frac{\gamma+\varepsilon}{\mathrm{J}}} .
$$

Focus attention that a weak discontinuity of temperature is not associated with the tangential projections of polarization vectors of weak discontinuities $A_{\perp}$ and $S_{\perp}$. 
In third case the propagation of wave surfaces of weak discontinuities displacements, microrotations and temperature is impossible if the constitutive characteristic of the type-II micropolar thermoelastic continuum does not satisfied certain limitations as determined by (32), i.e.

$$
G=c_{\perp}^{\mu}=c_{\perp}^{\mu \mu} .
$$

In fourth case the wave surface propagation is impossible if the normal projections of discontinuities polarization vectors are equal to zero. Thus equations (32) can be transformed into

$$
\left\{\begin{array}{l}
\left(\rho G^{2}-(\lambda+2 \mu)\right) A_{\|}-\alpha G B=0 \\
\left(\mathrm{~J} G^{2}-(\gamma+2 \beta)\right) S_{\|}-\varsigma G B=0 \\
B-\frac{\kappa}{\Lambda} G^{2} B+\frac{\alpha G}{\Lambda} A_{\|}+\frac{\varsigma G}{\Lambda} S_{\|}=0
\end{array}\right.
$$

There are only two cases of transverse wave propagation in MPTE-II continuum:

$$
\begin{aligned}
& \text { I case }-A_{\|} \neq 0, S_{\|} \neq 0, B \neq 0 ; \\
& \text { II case }-A_{\|} \neq 0, S_{||} \neq 0, B=0 .
\end{aligned}
$$

In first case we can easy obtained bicubic equation for normal wave velocity as follow

$$
\rho\left(\Lambda-\kappa G^{2}\right)+\frac{\alpha G^{2}}{G^{2}-c_{\|}^{2}}+\frac{\varsigma G^{2}}{G^{2}-c_{\|}^{\mu \mu 2}}=0,
$$

Here we denotes velocities by

$$
c_{\|}^{\mu \mu}=\sqrt{\frac{\beta+2 \gamma}{\mathrm{J}}}, \quad c_{\|}=\sqrt{\frac{\lambda+2 \mu}{\rho}} ;
$$

In second case the propagation of weak discontinuities surfaces is impossible if the constitutive characteristic of the type-II micropolar thermoelastic continuum does not satisfied certain limitations as determined by (32), i.e.

$$
G=c_{||}=c_{\|}^{\mu \mu},
$$

and for normal projection of discontinuities polarization vectors

$$
\frac{\alpha}{\varsigma}=-\frac{S_{\|}}{A_{\|}}
$$

\section{Conclusions}

In the present study problems of propagating surfaces of discontinuities of displacements, microrotation and thermal displacements of the micropolar type-II thermoelastic media (GNII) has been considered. The dynamical and constitutive equations for hyperbolic thermoelastic type-II micropolar continuum have been derived by field theory technique and formalism of the variational calculus. The special form of the first variation of the action integral has been used us in order to obtained 4-covariant jump conditions on wave surfaces.
The three-dimensional form of the jump conditions on the surface of a strong discontinuity of field can be derived as the result of its four-dimensional co- variant form. The possible normal velocities of the prop-agating surfaces of weak discontinuities are found. The possible normal velocities of the propagating surfaces of weak discontinuities have been found. The distinguished cases of propagating wave surfaces in MPTE-II continuum have been separately considered. Propagating surfaces of weak discontinuities have been discriminated depending on the spatial orientations of polarization vectors of discontinuities.

\section{Acknowledgements}

The authors wish to thank Professor Yu. N. Radayev for the statement of the problem, scientific advice and necessary support. This work was financially supported by the Russian Science Foundation under Grant 14-19-01280.

\section{References}

1. E. Cosserat and F. Cosserat, Theories of Deformable Bodies (Scientific Library A. Hermann et Fils, Paris, 1909) 242

2. W. Nowacki Theory of Asymmetric Elasticity (Pergamon Press, Oxford, 1986) 384

3. R. D. Mindlin and H. F. Tiersten Arch. for Rat. Mech. and Anal., 11:1 415-448 (1962)

4. A. V. Manzhirov and D. A. Parshin, Mechanics of Solids. 50 (5) 559-570 (2015)

5. A. V. Manzhirov and D. A. Parshin, Mechanics of Solids. 50 (6) 657-675 (2015)

6. A. C. Eringen, Microcontinuum field theories. Vol. 1. Foundations and Solids. (Springer, Berlin; Heidelberg; N.Y., 1999) 325

7. H. Ramézani, A. El-Hraiech, and J. Jeong, Comput. Methods Appl. Mech. Engrg. 237-240 227-243 (2012)

8. S. Chandrasekhar Liquid Crystals (Cambridge University Press, Cambridge, 1992) 460

9. T. F. McNelly et al. Phys. Reviews. 24:3 100-102 (1970)

10. Jackson H. E.,Walker C. T., and McNelly T. F. Phys. Reviews. 25:1 26-28 (1970)

11. Rogers S. J. Phys. Review B. 3:4 1440-1457 (1971)

12. Pohl D. W. and Irniger V. Phys. Review Letters. 36:9 480-483 (1976)

13. R.J. Hardy and S.S. Jaswal Phys. Review B. 3:12 4385-4387 (1971)

14. Narayanamurti V. and Dynes R. C. Phys. Reviews. 28 1461-1464 (1972)

15. V. A. Kovalev, E. V. Murashkin, and Yu. N. Radayev, Izv. Sarat. Univ. (N.S.), Ser. Mat. Mekh. Inform. 141 77-87 (2014).

16. V. A. Kovalev, E. V. Murashkin, and Yu. N. Radayev, Izv. Sarat. Univ. (N.S.), Ser. Mat. Mekh. Inform. 151 79-89 (2015).

17. A. E. Green and P. M. Naghdi, J. Therm. Stress, 15 253-264 (1992) 
18. A. E. Green and P. M. Naghdi, J. Elasticity.. 31 189$208(1993)$

19. E. V. Murashkin and Y. N. Radayev, Materials Physics and Mechanics, 23 10-13 (2015)

20. E. Murashkin and M. Polonik, On Compatibility Conditions for Propagating Surfaces with Additional Constraints. IUTAM Symposium on Growing solids. Symposium materials, 79-80, 2015.
21. V. A. Kovalev and Yu. N. Radayev, Wave Problems of Field Theory and Thermomechanics (Saratov. Univ. Pub., Saratov, 2010) 328

22. T. Y. Thomas, Plastic Flow and Fracture in Solids (Academic Press, N.Y., 1961) 271

23. W. J. M. Rankine, Proc. of the Royal Society of London. London: The Royal Society, 18 80-83 (1870)

24. P. H. Hugoniot, J. Ecole Polytechnique, CLVII 3-98 (1887) 